\title{
Magnetic Field Tunable Capacitive Dielectric:Ionic-liquid Sandwich Composites
}

$\mathrm{Ye} \mathrm{Wu}$

The University of Texas at San Antonio, Department of Electrical and Computer Engineering, One UTSA

Circle, San Antonio, TX 78249.

Abstract: We examined the tunability of the capacitance for $\mathrm{GaFeO}_{3}$-ionic liquid- $\mathrm{GaFeO}_{3}$ composite material by external magnetic and electric field. Up to 1.6 folds of capacitance tunability could be achieved at $957 \mathrm{kHz}$ with voltage $4 \mathrm{v}$ and magnetic field $0.02 \mathrm{~T}$ applied. We show that the capacitance enhancement is due to the polarization coupling between dielectric layer and ionic liquid layer.

PACS codes: 81

Keywords: magnetocapacitance,ionic liquid, capacitance enhancement

\section{INTRODUCTION}

There could be two effective strategies for strengthening the electro-magnetic coupling in materials.

One strategy is the introduction of magnetic ion dopant to make nonmagnetic electric materials magnetic. This is shown in the synthesis of room temperature ferromagnetic or antiferromagnetic semiconductors,[1]-[5] which is made by dopping magnetic particles into III-V semiconductors. Due to the low solubility of magnetic particles in the semiconductor composites, the electro-magnetic effects is relatively small.[1]

Another strategy is to reconstruct electric counterparts of conventional magnetic compound. This involves the reconstruction of the charge density and thus the electrical modification of the electric polarization in the magnetic material system. One approach could be integrating guest material which contains high carriers concentration. One candidate for this approach could be the ionic liquid, a 
conductive fluid which has been intensively used for carriers engineering of strongly correlated electron system. For example, it has been used for introducing novel superconducting states, achieving electroresistance tunability. [6]-[14]. In this letter, we sandwiched the ionic liquid between two $\mathrm{GaFeO}_{3}$ layers, a well known multiferroic materials.[15]-[21]

We performed the study of dielectric response of the $\mathrm{GaFeO}_{3}$-ionic liquid- $\mathrm{GaFeO}_{3}$ composite material under external magnetic field and bias voltage. We expect this composite material could hold the electro-magneto-dielectric properties. The motivation for this strategy is to find out the highly tunable dielectric response of composite material with respect to the external field, which is crucial for possible future applications in tunable microwave devices.

\section{Experiments.}

The $\mathrm{GaFeO}_{3}$ thick film samples(110nm) used in the study were produced by polymer assisted deposition method.[22] The $\mathrm{GaFeO}_{3}$ phases were confirmed by x-ray powder diffraction. The phases was confirmed by XRD (Figure 1(a)). The structure of $\mathrm{GaFeO}_{3}$ was refined and drawn by GSAS software package. [23][24] Then the refined unit cell is drawn by DRAWxtl.[25] One drop of ionic liquid 1-n-Butyl-3-methylimidazo-li-umhexafluorophosphate (Alfa Aesar) was placed between two slides of $\mathrm{GaFeO}_{3}$ and mechanically pressed to have a thickness around $100 \mu \mathrm{m}$. This gave us the $\mathrm{GaFeO}_{3}$-ionic liquid- $\mathrm{GaFeO}_{3}$ sandwich structure. The electric resistivity was measured by a standard 4 probes measurement setup equipped with an oven. The temperature hadn't been increased to higher than $398 \mathrm{~K}$ for sample damage concern. The magnetic field strength up to $21 \mathrm{~A} / \mathrm{m}$ was generated by a DC magnet. A dc power source( Agilent E3648A) was used for applying external bias voltage. We measured the capacitance using a LCR meter (Hp HEWLETT PACKARD 4284A).In order to eliminate possible leakage current from power source, the sample was placed between two 
commercial capacitors which provide isolation. The measurement unit was further electrically shielded and guarded in order to prevent leakage current. The magnetic field direction is perpendicular to the sample surface. The capacitance of the $\mathrm{GaFeO}_{3}$-ionic liquid- $\mathrm{GaFeO}_{3}$ sandwich structure was measured with respect to the external magnetic field and under dc voltages.

\section{Theory.}

For the following, it would be important to explore the nature of magento-electro-dielectric coupling in this composite material. Since it contains the bulky ionic liquid layer and the magnetic layer, we may suppose the total electric polarization is the superposition of the two kinds of polarization generated from these two distinct layers. This assumption is based on our dielectric measurement of ionic liquid and $\mathrm{GaFeO}_{3}$ material. The dielectric response of either $\mathrm{GaFeO}_{3}$ or ionic liquid doesn't show any distinct characterization when electric field or magnetic field is applied.

We suppose there are three major kinds of electric polarization in this composite material. The first is the polarization $\mathrm{P}_{1}$ that is generated from the dielectric layer of $\mathrm{GaFeO}_{3}$. The second is $\mathrm{P}_{2}$ from the ionic liquid layer and the third is from the interface polarization between the $\mathrm{GaFeO}_{3}$ and ionic liquid layer, which could be represented by $P_{12}=C P_{1}+\varepsilon_{0} E_{1}=C P_{2}+\varepsilon_{0} E_{2}$.

Here $\mathrm{C}$ represents the coupling factor[26] between the $\mathrm{GaFeO}_{3}$ and ionic liquid layer. $\mathrm{E}_{1}$ and $\mathrm{E}_{2}$ are the local electric field which could be fitted from the experimental data. The total polarization is $P=\lambda_{1} P_{1}+\lambda_{2} P_{2}+\lambda_{3}$

where $\lambda_{1}, \lambda_{2}$ and $\lambda_{3}$ are the constants which could be fitted from the experimental data. $P_{12}$ is not shown in this expression, since it could be also represented by $\mathrm{P}_{1}$ or $\mathrm{P}_{2}$ in Eq.(3.1) .

$\mathrm{P}_{1}$ could be calculated using classical method,[27] $\mathrm{P}_{1}=\frac{4 \pi\left(E-C_{\omega} \sum C_{\beta} H^{\beta}\right)}{\varepsilon_{0}}$

where $\mathrm{C}_{\omega}$ is the parameter which depends on the electric signal frequency. $\mathrm{C}_{\beta}$ is the constant that is 3 
associated with $\Sigma \mathrm{H}^{\beta}$. E is the electric field across the sample.

In the following, we will calculate $\mathrm{P}_{2}$. This calculation of the second kind of polarization could be started from the analysis of ions dynamic with respect to the magnetic field.

The ionic liquid would involve with the process of the exchange of two ions, which is actually the replacement of an anion by a cation. [28][29]We consider an anion in position A is replaced by a cation in position B. For simplicity, the velocity of cation from A to B is supposed to be the same with that of anion from B to $\mathrm{A}$. The charge $Q$ would undergo a magnetic force $Q H v$ and travel with a certain distance of $x$.

The work required in this exchange is

$W=(-Q)\left(\varphi_{A}-\varphi_{B}\right)+Q\left(\varphi_{B}-\varphi_{A}\right)+2 Q H v_{A B} x_{A B}=2 Q\left(\varphi_{B}-\varphi_{A}\right)+2 Q H v x$,

Where $2 Q H v x$ is the work attributed to the magnetic force.

Using Boltzmann's law, the equilibrium ionic concentrations at the two spots are in the ratio of

$\frac{c_{B}}{c_{A}}=\exp \left(\frac{-W}{k T}\right)=\exp \left[\frac{2 Q\left(\varphi_{A}-\varphi_{B}\right)-2 Q H v x}{k T}\right]$

We assume the total ionic concentration is constant, which is $c_{B}+c_{A}=c$.

Using Eq.(3.5) and Eq.(3.6), we can derive $c_{A}=\frac{c}{1+\exp (t)}$, (3.7) $c_{B}=\frac{c \exp (t)}{1+\exp (t)}$, (3.8)where $t=\frac{2 Q\left(\varphi_{A}-\varphi_{B}\right)-2 Q H v x}{k T}=\frac{2 Q \varphi(x)-2 Q H v x}{k T}$.

The Poission's law indicates $\frac{d^{2} \varphi(x)}{d x^{2}}=\frac{-\rho(x)}{\varepsilon}$.

Here the charge density could be expressed as $\rho(x)=F\left(c_{B}-c_{A}\right)=\frac{F(\exp (t)-1) c}{1+\exp (t)}$.

$F$ is the Faraday constant.

Plugging Eq.(3.9) and Eq.(3.11) into Eq.(3.10), we can generate $\frac{d^{2} t}{d x^{2}}=\frac{-F c}{\varepsilon} \frac{\exp (t)-1}{\exp (t)+1}$, 
which could be solved as $\frac{d t}{d x}=\sqrt{\frac{F_{c}}{2 \varepsilon}\left[t-2 \ln \left(1+e^{t}\right)\right]}$.

We suppose the charge on the dielectric surface is $q$, then the charge in the diffuse layer would be $-\mathrm{q}$ when the electroneutrality is concerned. It should be noted that

$-q=\int_{x}^{\infty} \rho(l) d l$.

Combining Eq.(3.11) and Eq.(3.14), we can get $q=\sqrt{2 F c \varepsilon\left[t-2 \ln \left(1+e^{t}\right)\right]}$

Using Eq.(3.13),we can have

$\frac{d t}{d x}=\sqrt{\frac{F_{c}}{2 \varepsilon}\left[t-2 \ln \left(1+e^{t}\right)\right]}=\frac{q}{2 \varepsilon}$

Then the polarization could be calculated as

$$
P_{2}=\gamma \frac{\partial q}{\partial \varphi}=\gamma \frac{\partial q}{\partial t} \frac{\partial t}{\partial \varphi}=\frac{\gamma \sqrt{2 F c \varepsilon} Q\left(1-e^{t}\right)}{k T \sqrt{t-2 \ln \left(1+e^{t}\right)}\left(1+e^{t}\right)}=\frac{\gamma \sqrt{2 F c \varepsilon} Q\left(1-e^{t}\right)}{k T \sqrt{t}\left(1+e^{t}\right)}
$$

where $\gamma=d E_{0} / A$ is the constant that associated with sample dimension $d$, sample area $A$ and localized field $E_{0}$.

Combining Eq.(3.3) and Eq.(3.17), the total capacitance of dielectric: ionic structure is calculated as

$$
C_{t o t}=P / \gamma=\frac{4 \pi \lambda_{1}\left(E-C_{\omega} \sum C_{\beta} H^{\beta}\right)}{\varepsilon_{0} \gamma}+\frac{\lambda_{2} \sqrt{2 F c \varepsilon} Q\left(1-e^{t}\right)}{k T \sqrt{t}\left(1+e^{t}\right)} .
$$

\section{Results and analysis.}

Fig.A1(a) (listed in Appendix A)shows the XRD result of $\mathrm{GaFeO}_{3}$ film. We used GSAS package to refine the structure. The lattice length was refined as, $a=8.751 \AA, b=9.398 \AA, c=5.079 \AA$. The lattice angles are $\alpha=\beta=\gamma=90^{\circ}$. The space group is Pc21n. As shown in Fig.A1(b)(Appendix A), every gallium atom is located at the center of octahedron formed by six ozone atoms. We measure the resistance dependence on temperature. When $295 \mathrm{~K}<\mathrm{T}<388 \mathrm{~K}$, this $\mathrm{GaFeO}_{3}:$ ionic liquid sandwich structure shows quasi-semiconductor transport (Fig.A1(c)). 
It should be noted that the electrochemical window of the ionic liquid is in the range not higher than than $6 \mathrm{v}$. For this reason, the external voltage applied was only $4 \mathrm{v}, 5 \mathrm{v}$ and $6 \mathrm{v}$, respectively, which could make physical and chemical properties of the ionic liquid stable.

In order to investigate the electro-magneto-dielectric properties of this composite material, we investigated the capacitance tunabilities corresponding to varied electric frequencies, voltages and magnetic field (Fig.A2). The capacitance tunabilities in Fig.A2 are defined as $\Delta \mathrm{C}_{\mathrm{C}}$, where $\mathrm{C}_{0}$ is the origin capacitance with magnetic field $\mathrm{H}=0.0069 \mathrm{~T} . \Delta \mathrm{C}$ is the variation of capacitance corresponding to $\mathrm{C}_{0}$. It shows that the capacitance tunability with voltage $4 \mathrm{v}$ is relatively higher than those with voltage $0 \mathrm{v}, 5 \mathrm{v}$ and $6 \mathrm{v}$. This result indicates the $4 \mathrm{v}$ may be the optimized bias voltage. $160 \%$ capacitance tunability could be achieved at the frequency $957 \mathrm{kHz}$ with the voltage $4 \mathrm{v}$ and the magnetic field $0.02 \mathrm{~T}$.

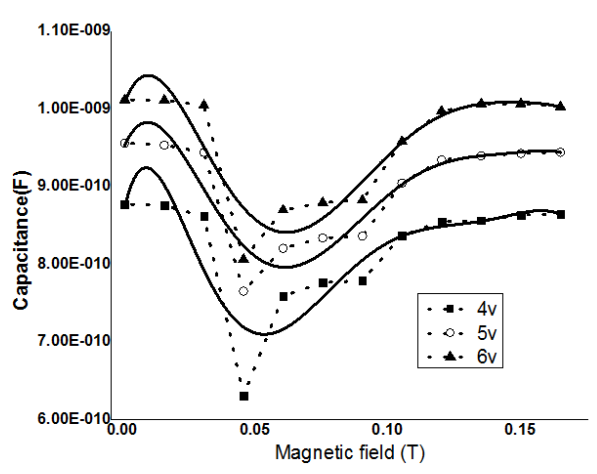

Figure 1 Comparing the experimental and simulated data of magnetocapacitance for the $957 \mathrm{k} \mathrm{Hz}$ and with varied voltage and magnetic field applied.( The dash lines represent the experimental results while the solid lines represent the simulated results)

Figure 1 compares the experimental and simulated data of the capacitance with fixed voltage of $4 \mathrm{v}, 5 \mathrm{v}$ and $6 \mathrm{v}$ and with varied magnetic field. The order of the capacitance dependence on the magnetic field $\beta$ (Eq.(3.19))was fitted as 7, which suggested a very strong coupling between the magnetic field and polarization. 
It should be noted that the experimental data corresponding to $957 \mathrm{~Hz}$ in fig. 1 was actually picked up from one of the data points in fig.A2(b). It would be cumbersome if we have to show all the simulated data corresponding to different frequency. For this reason we only choose the presentation of the experimental and simulated data corresponding to one specific frequency of $957 \mathrm{~Hz}$. The other results with other frequency are similar, which all show nonlinear dependence of the capacitance on the magnetic field. In closing, we do show a very precise quantitative analysis of our data in terms of theoretical calculations capturing all the details of the composite material investigated in the present scope; we also show the trend of transition and predict the trend of the capacitance dependence on the external magnetic field and voltage. This simple model simulates the important characteristics of capacitance with varied magnetic field and electric field in this composite material.

\section{CONCLUSION.}

$\mathrm{GaFeO}_{3}$-ionic liquid- $\mathrm{GaFeO}_{3}$ composite material shows quasi-semiconductor transport when $295 \mathrm{~K}<\mathrm{T}<388 \mathrm{~K}$. We examined the electro-magneto-dielectric effect on this structure. We find that up to 1.6 folds capacitance tunability could be achieved at $975 \mathrm{kHz}$ with the interaction of the magnetic field $0.02 \mathrm{~T}$ and bias voltage $4 \mathrm{v}$. We present a mechanism which takes account of the coupling between the ionic liquid layer and the solid dielectric layer. The effect shows a possible route to use the ionic liquid integrated with the dielectric material and suggests a simple way to introduce tunable dielectric permittivity.

\section{ACKNOWLEDGMENTS}

The authors thank Prof.Robert Whetten in UTSA Physics Department for many useful discussions. This work was supported by the National Science Foundation under the Grants ECCS \#1002380 and DMR \#0844081. 


\section{Appendix A:}

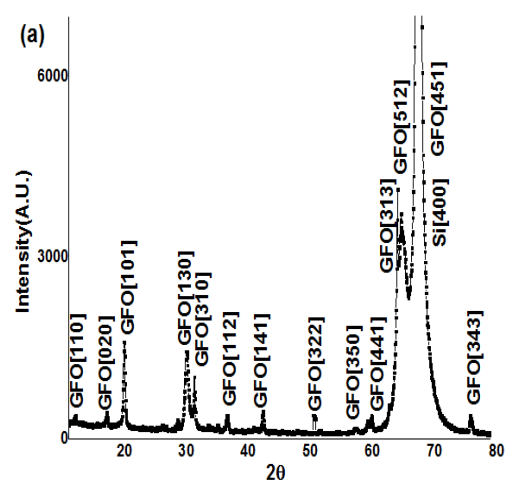

(b)

(c)

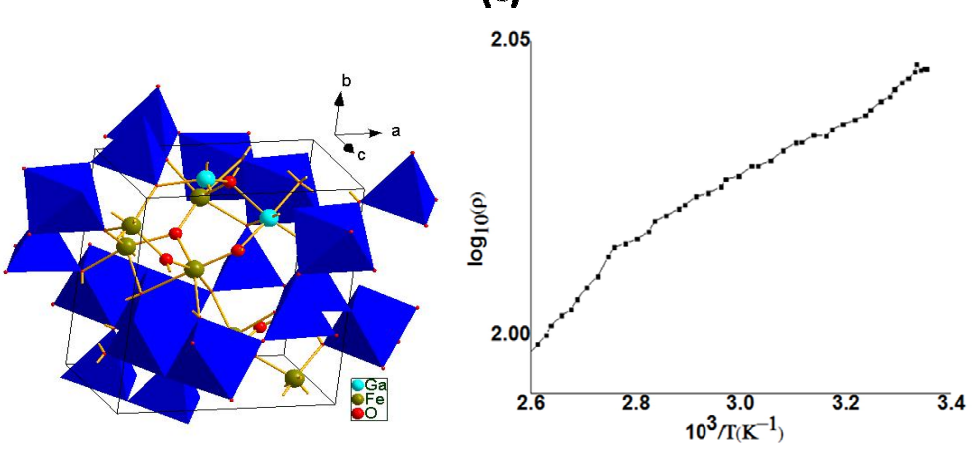

Figure A1.(a)XRD profile of $\mathrm{GaFeO}_{3}$.(b)the unit cell of $\mathrm{GaFeO}_{3}$.(c) Resistance vs. temperature characterization of the $\mathrm{GaFeO}_{3}$ : ionic liquid sandwich structure.
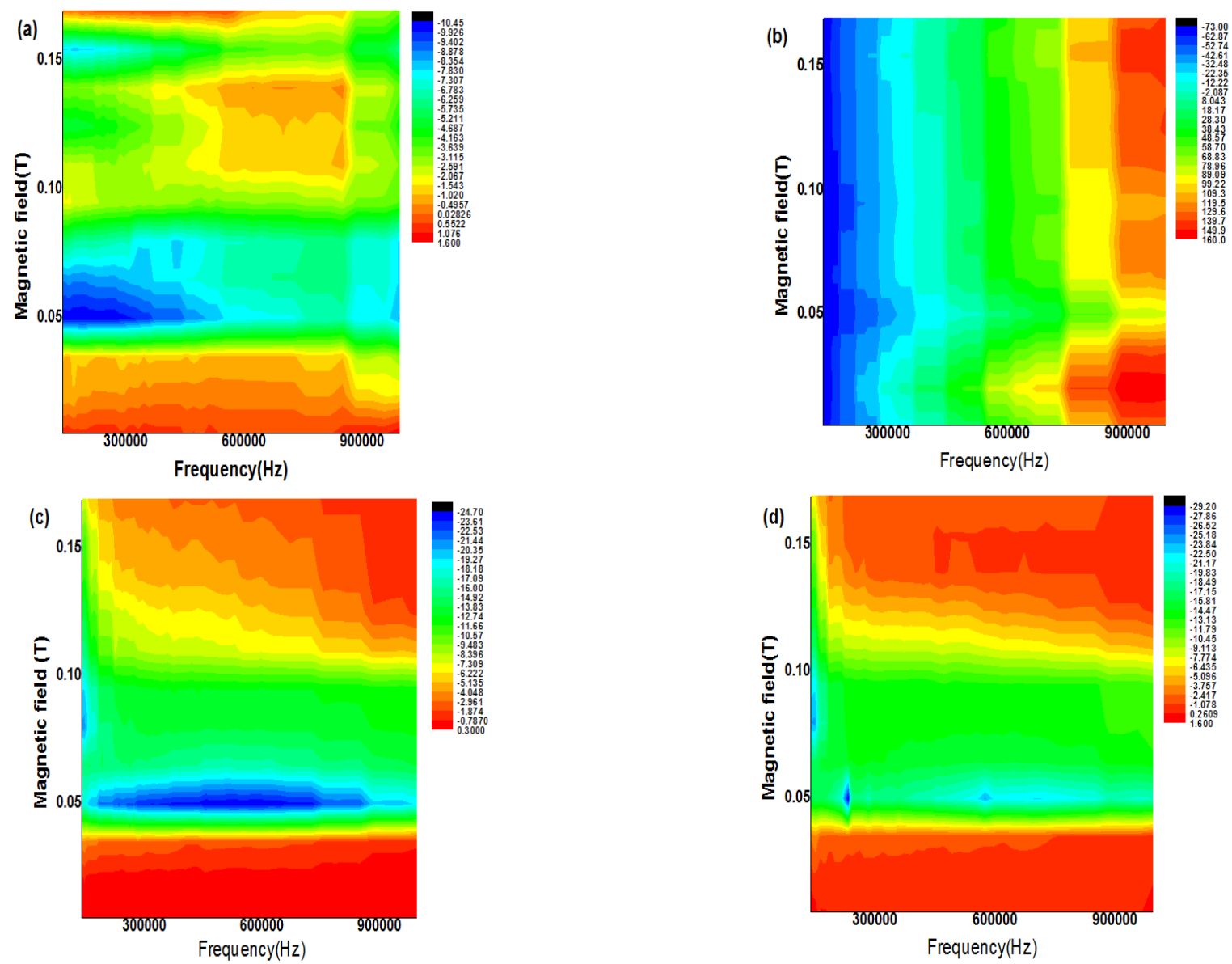

Figure A 2 . 2D capacitance tunability mapping corresponding to different bias voltage (a) $0 \mathrm{v}$ (b) $4 \mathrm{v}$

(c) $5 \mathrm{v}(\mathrm{d}) 6 \mathrm{v}$.

\section{References:}


[1] Ohno H: Making nonmagnetic semiconductors ferromagnetic. Science.1998; 281: 951.

[2] Matsumoto $\mathrm{Y}$ et al. : Room-temperature ferromagnetism in transparent transition metal-doped titanium dioxide. Science $.2001 ; 291: 854$.

[3] Nguyen HH, Sakai J, Poirot N and Brize V: Room-temperature ferromagnetism observed in undoped semiconducting and insulating oxide thin filmsPhys. Rev. B. 2006; 73: 132404.

[4] Pan H et al. : Room-Temperature Ferromagnetism in Carbon-Doped ZnO. Phys.Rev.Lett. 2007;99 : 127201.

[5] Marti X et al. : Room-temperature antiferromagnetic memory resistor.Nature Materials 2014; 13:367.

[6]Zhang Y, Jianting Y, Yusuke M, and Yoshihiro I: Ambipolar MoS2 Thin Flake Transistors.Nano Lett. 2012;12: 1136.

[7] Ueno $\mathbf{K}$ et al.: Discovery of superconductivity in $\mathrm{KTaO}_{3}$ by electrostatic carrier doping.

Nature Nanotechnology.2011; 6:408.

[8] Hiroyasu $\mathrm{N}$ et al. :Electroresistance Effect in Gold Thin Film Induced by Ionic-Liquid-Gated Electric Double Layer. Appl.Phys.Exp. 2012;5: 023002.

[9] Kasahara Y et al.: Electric-Field-Induced Superconductivity Detected by Magnetization Measurements of an Electric-Double-Layer Capacitor.J. Phys. Soc. of Jpn. 2012;80: 023708.

[10]Ye $\mathbf{J}$ et al.: Accessing the transport properties of graphene and its multilayers at high carrier density .Proc. of Nat. Aca. Sci. 2011;108: 13002.

[11]Ye $\mathrm{J}$ et al.:Liquid-gated interface superconductivity on an atomically flat film. Nature materials.2010; 9:125.

[12]Ye J et al. : Superconducting Dome in a Gate-Tuned Band Insulator. Science.2012; 338:1193. 
[13]Yuan $\mathrm{H}$ et al. : Electrostatic and Electrochemical Nature of Liquid-Gated Electric-Double-Layer Transistors Based on Oxide Semiconductors.J. of Am. Chem. Soc. 2010;132:18402.

[14] Ueno K et al., Field-Induced Superconductivity in Electric Double Layer Transistors.J. Phys. Soc. Jpn. 2014;83: 032001.

[15]Remeika JP: GaFeO3: A Ferromagnetic-Piezoelectric Compound. J. Appl. Phys.1960; 31: S263.

[16]M.B.Mohamed et al.:Dielectric relaxation and magnetic properties of $\mathrm{Cr}$ doped GaFeO3. J. Phys.

D: Appl. Phys. 2010;43: 455409.

[17]Saha R et al. :Phase Transitions of $\mathrm{AlFeO} 3$ and $\mathrm{GaFeO} 3$ from the Chiral Orthorhombic (Pna21) Structure to the Rhombohedral (R3c) Structure.Inorg. Chem. 2011;50:9527.

[18]Saha $\mathrm{R}$ et al.: Multiferroic and magnetoelectric nature of $\mathrm{GaFeO}_{3}, \mathrm{AlFeO}_{3}$ and related oxides. Solid State Comm. 2012;152:1964.

[19]Mukhopadhyay K et al.: Enhanced Magnetic Behavior of Chemically Prepared Multiferroic Nanoparticles of $\mathrm{GaFeO} 3$ in $(\mathrm{GaFeO} 3) 0.50$ (Ni0.4Zn0.4Cu0.2 Fe2O4)0.5 Nanocomposite.J. Phys. Chem. C 2012;116:4948.

[20]Bahoosh SG and Wesselinowa JM:Origin of the different multiferroism in $\mathrm{BiFeO}_{3}$ and $\mathrm{GaFeO}_{3}$.J.of Appl. Phys. 2013;113: 063905.

[21]Mukherjee $\mathrm{S}$ et al.: Room Temperature Nanoscale Ferroelectricity in Magnetoelectric $\mathrm{GaFeO}_{3}$ Epitaxial Thin Films. Phys. Rev. Lett. 2013;111: 087601.

[22]Jia QX et al. :Polymer-assisted deposition of metal-oxide films. Nature Materials 2004; 3: 529.

[23]Larson ACand Von Dreele RB: GENERAL STRUCTURE ANALYSIS SYSTEM .Los Alamos National Laboratory Report LAUR .1994; 86-748.

[24]Toby BH: EXPGUI, a graphical user interface for GSAS.J. Appl. Cryst. 2001;34:210-213. 
[25]Finger LW et al.: DRAWxtl, an open-source computer program to produce crystal structure drawings. J. Applied Crystallography.2007;40:188-192.

[26]Okatan MB et al.: Polarization coupling in ferroelectric multilayers. Phys.Rev.B 2009;79: 174113. [27]Wu Y, Guo R, Bhalla A: Magneto-resistance and -capacitance dual tunability of $\mathrm{SmFeO}_{3}$. ( in preparation)

[28]Kornyshev AA : Double-Layer in Ionic Liquids: Paradigm Change?J. Phys. Chem. B.2007; 111: 5545.

[29]Oldham KB :A Gouy-Chapman-Stern model of the double layer at a (metal)/(ionic liquid) interface. J. of Electroanalytical Chem. 2008;613:131. 